\title{
CORRECTIONS APPLIED TO THE CARRIER FREQUENCIES OF The \\ RADIO STATIONS GBR AND RES ACCORDING TO THE USSR \\ STATE STANDARD OF TIME AND FREQUENCY
}

TABLE 1. Corrections applied to the Carrier Frequencies of the Radio Stations GBR and RES According to the USSR State Standard of Time and Frequency

\begin{tabular}{|c|c|c|c|c|c|c|c|c|}
\hline \multirow[b]{2}{*}{ date } & \multicolumn{8}{|c|}{ January, 1971} \\
\hline & $\begin{array}{c}\text { GBR' } 16 \mathrm{kHz} \\
\text { referred to } \\
03^{\mathrm{h}} \mathrm{TU} \\
\end{array}$ & $\begin{array}{c}\mathrm{RES}, 100 \mathrm{kHz} \\
\text { referred to } \\
19^{\mathrm{h}} \mathrm{TU}\end{array}$ & date & $\begin{array}{r}\mathrm{GBR}, 16 \mathrm{kHz} \\
\text { referred to } \\
03^{\mathrm{h}} \mathrm{TU}\end{array}$ & $\begin{array}{c}\text { RES, } 100 \mathrm{kHz} \\
\text { referred to } \\
19^{\mathrm{h}} \mathrm{TU} \\
\end{array}$ & date & $\begin{array}{c}\text { GBR, } 16 \mathrm{kHz} \\
\text { referred to } \\
03^{\mathrm{h}} \mathrm{TU} \\
\end{array}$ & $\begin{array}{c}\text { RES } 100 \mathrm{kHz} \\
\text { referred to } \\
19^{\mathrm{h}} \mathrm{TU}\end{array}$ \\
\hline $\begin{array}{r}1 \\
2 \\
3 \\
4 \\
5 \\
6 \\
6 \\
7 \\
8 \\
9 \\
10\end{array}$ & $\begin{array}{l}-300,1 \cdot 10^{-10} \\
=300,0 \\
=300,1 \\
=300,2 \\
=300,1 \\
=300,5 \\
=299 \cdot 9 \\
-300,1 \\
-300,2\end{array}$ & $\begin{array}{l}-300,2 \cdot 10^{-10} \\
-300,6 \\
=300 ; 4 \\
=300,3 \\
-300,5 \\
=299,9 \\
=301,0 \\
-301,0 \\
-300,4\end{array}$ & $\begin{array}{l}11 \\
12 \\
13 \\
14 \\
15 \\
16 \\
17 \\
18 \\
19 \\
20\end{array}$ & $\begin{array}{l}-300,1 \cdot 10^{-10} \\
-300,1 \\
-300,2 \\
-300,3 \\
-300,3 \\
=200,0 \\
=299,9 \\
=300,2 \\
=300,0 \\
-300,0 \\
-300,2\end{array}$ & $\begin{array}{l}-300,7 \cdot 10^{-10} \\
-300,2 \\
-300,0 \\
-299,6 \\
-299,4 \\
-299,4 \\
-290,8 \\
-300,2 \\
-299,5 \\
-299,8 \\
-299,9\end{array}$ & $\begin{array}{l}21 \\
22 \\
23 \\
24 \\
25 \\
26 \\
27 \\
28 \\
29 \\
30 \\
31\end{array}$ & $\begin{array}{l}-300,4 \cdot 10^{-10} \\
-300,4 \\
-299,9 \\
-300,0 \\
-300,0 \\
=300,2 \\
=350,1 \\
-300,1 \\
-299,9 \\
-300,2 \\
-300,0\end{array}$ & $\begin{array}{l}-299,8 \cdot 10^{-10} \\
-299,8 \\
-2999.9 \\
-299,8 \\
-299,7 \\
-299,7 \\
-29997 \\
-299,7 \\
=2999.7 \\
=2999,7 \\
-299,7\end{array}$ \\
\hline & & & & & & mean & $-300,1$ & $-300,0$ \\
\hline
\end{tabular}

Translated from Izmeritel'naya Tekhnika, No. 4, p. 96, A pril, 1970.

(1)1971 Consultants Bureau, a division of Plenum Publishing Corporation, 227 West 17th Street, New York, N.Y. 10011. All rights reserved. This article cannot be reproduced for any purpose whatsoever without permission of the publisher. A copy of this article is available from the publisher for $\$ 15.00$. 